\title{
NOVEL SYNTHESIS AND BIOLOGICAL ACTIVITY OF (2E)-1- (3-AMINO-5-CHLORO-1-BENZOFURAN-2-YL)-3-ARYLPROP- 2-EN-1-ONES AND THEIR DERIVATIVES
}

\author{
Mahesh Kumar $^{1}$, K. M. Basavaraja ${ }^{1,}$, Manjunatha Harihara Mathada ${ }^{2}$ \\ and M. Mylarappa ${ }^{3}$ \\ ${ }^{1}$ Department of Chemistry, Vijayanagara Sri Krishnadevaraya University, Ballari -583104, \\ Karnataka, India \\ ${ }^{2}$ SB Arts and KCP Science College, Vijayapura-586103, Karnataka, India. \\ ${ }^{3}$ Department of Chemistry, Sri Jagadguru Renukacharya College of Science, Arts \& Commerce, \\ Racecourse Road Bengaluru-560009, Karnataka, India. \\ ${ }^{\square}$ Corresponding Author: kmbasavaraja@gmail.com
}

\begin{abstract}
In continuation of our search for pharmacologically potent benzofuran compounds, we undertook the synthesis of 5chloro-2-hydroxy benzonitrile (2) from 5-chloroslicylaldehyde (1) by treating with acetic anhydride and sodium ethoxide. We get biologically active benzofurans and their derivatives. When compound 2 was treated with chloroacetone, yielded compound 3. Then the compounds $\mathbf{4}$ and chalcones 5a-e were prepared by treating compound $\mathbf{3}$ with aqueous sodium hydroxide and substituted aldehyde, respectively. Compound 5a was converted into compound $\mathbf{6 a}$ by treating with acetic anhydride. All newly synthesized compounds have been characterized by IR, ${ }^{1} \mathrm{HNMR}$, and Mass spectral data. All the extracts were screened for antimicrobial activities against various pathogenic microorganisms and exhibited appreciable activities compared with standard drugs.
\end{abstract}

Keywords: Benzofurans, Chalcones, Antibacterial, Antifungal Studies.

RASĀYAN J. Chem., Vol. 14, No.3, 2021

\section{INTRODUCTION}

Simple to complex structures of Chalcones (1,3-diaryl-2-propen-1-ones) have produced significant research over the last few decades. These act as precursors intermediates for synthesizing beneficial biologically active heterocyclic compounds such as pyrimidine, isoxazole, pyrazole, benzofuranone, quinolinone, etc. The existence of three carbon unsaturated between two aromatic rings shows an extensive biological studies such as anti-invasive activity, anti-HIV, anti-inflammatory, anti-malaria, anti-cancer and antibacterial activities. ${ }^{1-9}$ Benzofurans derivatives are of great interest in medicinal chemistry as a result of their therapeutic activity. ${ }^{10}$ This is the first work we have read in the scientific literature that supports the notion that Benzofuran-substituted Chalcones derivatives have anticancer properties. $^{11}$ Many new benzofuran compounds with therapeutic properties are currently under development. ${ }^{12}$ These many molecules have been synthesized in different ways. In this route, relatively simple Benzofuran molecules are obtained from which pharmacologically active molecules can be constructed. The current route is widely used to synthesize pharmacologically potent benzofuran heterocycles have shown moderate to good activity against various types of organisms. ${ }^{13,14}$

The present work reveals the compound 5-chloro-2-hydroxybenzonitrile 2 from 5-chloroslicylaldehyde (1) by treating acetic anhydride and sodium ethoxide. We have continued to get biologically active benzofurans and their derivatives. Compound 2 was treated with chloroacetone to yield compound 3. Then the compound N-(2-Acetyl-5-chloro-2, 3-dihydro-1-benzofuran-3-yl) acetamide (4) and Chalcones (2E)-1-(3-Amino-5-chloro-1-benzofuran-2-yl)-3-arylprop-2-en-1-ones 5 (a-e) were obtained by treating with aqueous sodium hydroxide and various substituted aldehyde, respectively. Further, compound 5a was converted into its acetyl derivative $\mathbf{6 a}$ by treating with acetic anhydride. This proves that the amino group at 3 - position is free to explore the scope of different applications. 


\section{Material and Methods}

\section{EXPERIMENTAL}

The materials were purchased from analytical quality grade without further purification. Melting points are reported at degrees Celsius. They were determined in open capillary tubes (using the Thiele tube) and are not corrected. FTIR-Perkin Elmer Spectrum-Two spectrophotometers (range: (IR) 4000-400 $\mathrm{cm}^{-1}$ ) are used to record IR spectrum (KBr disc method). ${ }^{1} \mathrm{HNMR}$ spectra were recorded on BRUKER $400 \mathrm{MHz}$ and ECX-JEOL 400(S), AVIII400(L) using $\mathrm{CDCl}_{3}$ and $\mathrm{DMSOD}_{6}$ as TMS solvents an internal standard. Mass Spectra were recorded in water model-synapt G2, APCI source Positive mode, Desolvation 200 Lit/hr.

Experimental Scheme: Include oxime step: 5-chlorosalicylaldehyde- $\rightarrow$ oxime
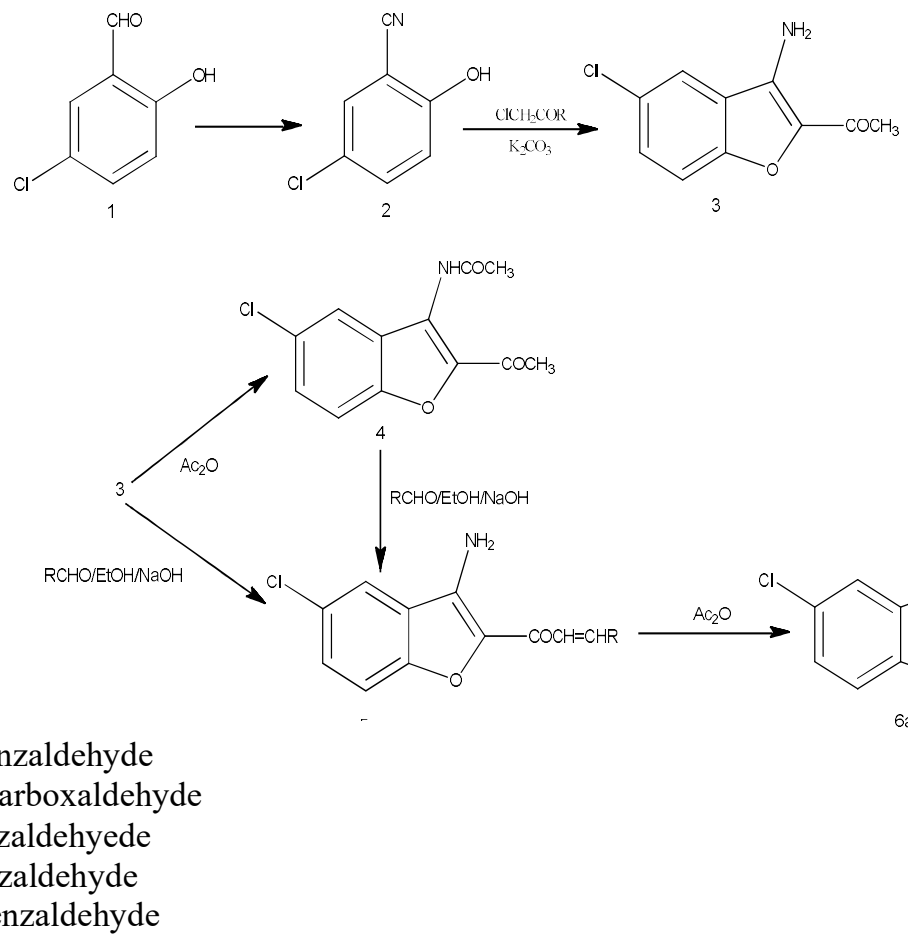

$\mathrm{R}, \mathrm{a}=4$-nitrobenzaldehyde

$\mathrm{b}=4$-pyridine carboxaldehyde

$\mathrm{c}=\mathrm{p}$-chlorobenzaldehyede

$\mathrm{d}=$ o-chlorobenzaldehyde

$\mathrm{e}=\mathrm{p}$-methoxybenzaldehyde

\section{Preparation of 5-chlorosalicylaldehyde}

Scheme-1

Conc. $\mathrm{HCl}$ was taken in a separating funnel and is added drop wise to $\mathrm{KMNO}_{4}$ contained in a receiver flask. Addition of conc. $\mathrm{HCl}$ was continued till the release of chlorine gas. The released gas was passed through the reagent bottle containing $250 \mathrm{ml}$ of conc. $\mathrm{H}_{2} \mathrm{SO}_{4}$ (as dehydrating agent). Dry chlorine gas was passed into a round-bottomed flask containing salicylaldehyde $(48.8 \mathrm{~g}, 0.4 \mathrm{~mol})$ with occasional stirring till saturation. The separated crystalline solid was collected and further purified by recrystallization with aqueous ethanol. The yield and melting point were recorded in Table-1.

\section{Preparation of 5-chlorosalicylaldehyde oxime}

5-Chloro Salicylaldehyde $(1.56 \mathrm{~g}, 0.01 \mathrm{~mol})$ and hydroxylamine hydrochloride $(0.70 \mathrm{~g}, 0.01 \mathrm{~mol})$ were dissolved in aqueous ethanol $(75 \mathrm{ml}$ ethanol and $50 \mathrm{ml}$ water). The solution was stirred constantly with the drop-wise addition of $\mathrm{NaOH}$ solution $(2.5 \mathrm{~g}$ in $5 \mathrm{ml}$ of water) by cooling under tap water. After the addition of $\mathrm{NaOH}$ is over, the reaction mixture was refluxed for half an hour, cooled and diluted with water.

\section{Preparation of 5-Chloro-2-hydroxybenzonitrile (2)}

Dry Salicylaldehyde oxime $(1.715 \mathrm{~g}, 0.01 \mathrm{~mol})$ and acetic anhydride $(1.02 \mathrm{~g}, 0.01 \mathrm{~mol})$ were refluxed for 1 hr. The acetic anhydride was removed under reduced pressure. The crude dark-colored intermediate 
(isoxazole) was treated with freshly prepared sodium ethoxide $(0.01 \mathrm{~mol})$. The obtained product was stirred for one hour at room temperature $\left(28^{\circ} \mathrm{C}\right)$. The reaction mixture was poured on crushed ice and carefully acidified with dilute $\mathrm{HCl}$. The title compound 2 was further purified by recrystallisation with ethanol. The yield and melting point were recorded in Table-1.

Table-1: Percentage Yield, Melting Point and Formula of 1, 2, 3, 4, (5a-e) and 6a

\begin{tabular}{c|c|c|c}
\hline Compounds & Yield (\%) & MP $\left({ }^{0} \mathrm{C}\right)$ & Molecular Formula \\
\hline 1 & 90 & 98 & $\mathrm{C}_{5} \mathrm{H}_{7} \mathrm{ClO}_{2}$ \\
\hline 2 & 85 & 118 & $\mathrm{C}_{7} \mathrm{H}_{4} \mathrm{ClNO}$ \\
\hline 3 & 78 & 158 & $\mathrm{C}_{10} \mathrm{H}_{10} \mathrm{ClNO}_{2}$ \\
\hline 4 & 95 & 92 & $\mathrm{C}_{12} \mathrm{H}_{12} \mathrm{ClNO}_{3}$ \\
\hline $5 \mathrm{a}$ & 98 & 144 & $\mathrm{C}_{19} \mathrm{H}_{13} \mathrm{ClN}_{2} \mathrm{O}_{5}$ \\
\hline $5 \mathrm{~b}$ & 95 & 138 & $\mathrm{C}_{19} \mathrm{H}_{15} \mathrm{ClN}_{2} \mathrm{O}_{3}$ \\
\hline $5 \mathrm{c}$ & 98 & 132 & $\mathrm{C}_{19} \mathrm{H}_{13} \mathrm{Cl}_{2} \mathrm{NO}_{3}$ \\
\hline $5 \mathrm{~d}$ & 90 & 129 & $\mathrm{C}_{19} \mathrm{H}_{13} \mathrm{Cl}_{2} \mathrm{NO}_{3}$ \\
\hline $5 \mathrm{e}$ & 92 & 162 & $\mathrm{C}_{20} \mathrm{H}_{14} \mathrm{ClNO}_{4}$ \\
\hline $6 \mathrm{a}$ & 90 & 198 & $\mathrm{C}_{19} \mathrm{H}_{14} \mathrm{ClNO}_{3}$ \\
\hline
\end{tabular}

Preparation of 1-(3-amino-5-chloro-2, 3-dihydro-1-benzofuran-2-yl) ethan-1-one (3)

A mixture of 5-Chloro-2-hydroxybenzonitrile (2) $(1.53 \mathrm{~g}, 0.01 \mathrm{~mol}), 50 \mathrm{ml}$ of dry acetone, $5 \mathrm{~g}$ of anhydrous potassium carbonate, and chloroacetone $(1.38 \mathrm{~g}, 0.015 \mathrm{~mol})$ was refluxed for 12 hours. Excess acetone was removed by distillation under reduced pressure. Solid potassium carbonate was filtered off to obtain the titled product 3 . The compound was further purified by recrystallisation from ethanol. The yield and melting point shall be recorded in Table-1.

\section{Preparation of N-(2-acetyl-5-chloro-2, 3-dihydro-1-benzofuran-3-yl) acetamide (4)}

A mixture of compound $3(0.01 \mathrm{~mol})$ and aqueous $\mathrm{NaOH}(10 \%, 30 \mathrm{ml})$ was treated with acetyl chloride $(10 \mathrm{ml})$ with continuous stirring conditions and kept at $28^{\circ} \mathrm{C}$ for $1 \mathrm{hr}$. The isolated solid was collected by filtration and washed with distilled water. Pure compound 4 was obtained by using ethanol for recrystallization. The yield and melting points were recorded in Table-1.

General procedure for the synthesis of (2E)-1-(3-Amino-5-chloro-1-benzofuran-2-yl)-3-arylprop-2en-1-ones (5a-e)

A mixture of compound $3(0.01 \mathrm{~mol})$ and appropriate aldehyde $(0.01 \mathrm{~mol})$ was dissolved in $50 \mathrm{ml}$ of ethanol and cooled to 5 to $10^{\circ} \mathrm{C}$. To this cooled aqueous $\mathrm{NaOH}$ solution $(5 \mathrm{ml}, 20$ percent) was slowly added. The mixture was stirred for two hours and kept overnight. The product was collected and recrystallized from ethanol. The yield and melting point are shown in Table-1.

\section{Preparation of $\mathrm{N}$-\{5-chloro-2-[(2E)-3-arylprop-2-enoyl]-1-benzofuran-3-yl $\}$ acetamide (6a)}

A mixture of compound $5 \mathrm{a}(0.01 \mathrm{~mol})$ and aqueous $\mathrm{NaOH}$ solution $(10$ percent, $30 \mathrm{ml})$ was treated with acetyl chloride $(10 \mathrm{ml})$ drop wise under stirring conditions and kept at room temperature for 1 hour. The separated solid was collected and washed with water. The pure 6a compound was obtained by recrystallization with ethanol. Yield and melting point shall be recorded in Table-1.

\section{FTIR, MASS and NMR Spectroscopic Studies}

\section{RESULTS AND DISCUSSION}

\section{Compound 3}

Obtained as a White solid; IR $\left(\mathrm{cm}^{-1}, \mathrm{KBr}\right): 1633(\mathrm{C}=\mathrm{O}), 3316\left(\mathrm{NH}_{2}\right) \cdot ;{ }^{1} \mathrm{H}-\mathrm{NMR}\left(\mathrm{CDCl}_{3}, 400 \mathrm{MHz}\right): \delta 3.50$ $\left(\mathrm{s}, \mathrm{CO}-\mathrm{CH}_{3}, 3 \mathrm{H}\right), 5.88\left(\mathrm{~s}, \mathrm{NH}_{2}, 2 \mathrm{H}\right), 6.58-6.80(\mathrm{~m}, \mathrm{Ar}-\mathrm{H}, 3 \mathrm{H})$, Mass $(\mathrm{m} / \mathrm{z})\left(\mathrm{M}^{+}\right): 210$.

\section{Compound 4}

Obtained as a White solid; IR $\left(\mathrm{cm}^{-1}, \mathrm{KBr}\right): 1674(\mathrm{C}=\mathrm{O}), 3306(\mathrm{NH}) ;{ }^{1} \mathrm{H}-\mathrm{NMR}$ (DMSO-d6, $400 \mathrm{MHz}$ ): $\delta$ $2.19\left(\mathrm{~s}, \mathrm{NHCOCH}_{3}, 3 \mathrm{H}\right), 2.53\left(\mathrm{~s}, \mathrm{COCH}_{3}, 3 \mathrm{H}\right), 7.59(\mathrm{~s}, \mathrm{NH}, 1 \mathrm{H}), 7.72-7.74(\mathrm{~m}, \mathrm{Ar}-\mathrm{H}, 3 \mathrm{H}) . \mathrm{Mass}(\mathrm{m} / \mathrm{z})$ $\left(\mathrm{M}^{+}\right): 251$. 
RASĀYAN J. Chem.

Vol. 14 | No. 3 |1699-1710| July - September | 2021

${ }^{1}$ H-NMR Spectra
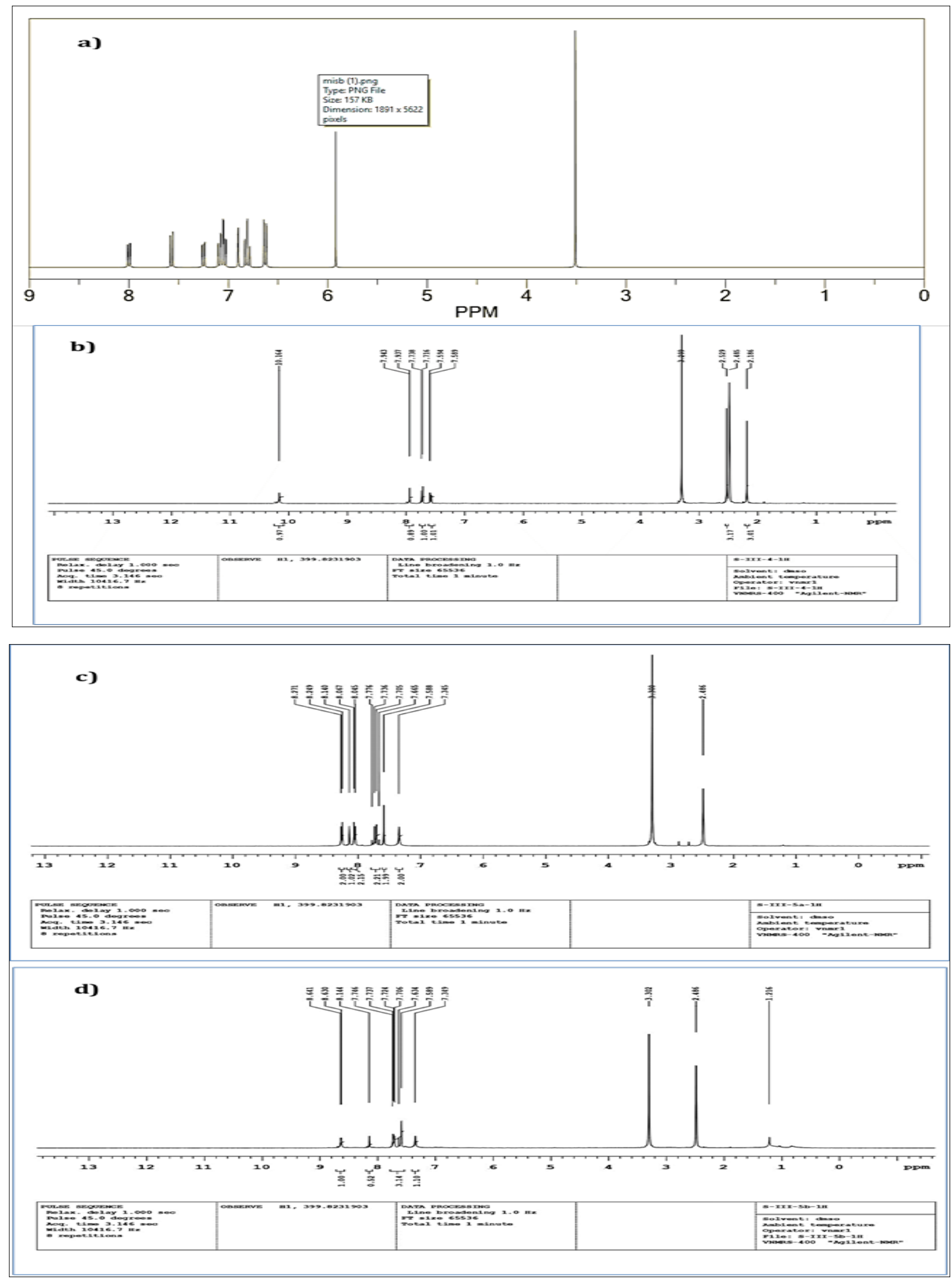
RASĀYAN J. Chem.

Vol. 14 | No. 3 |1699-1710| July - September | 2021
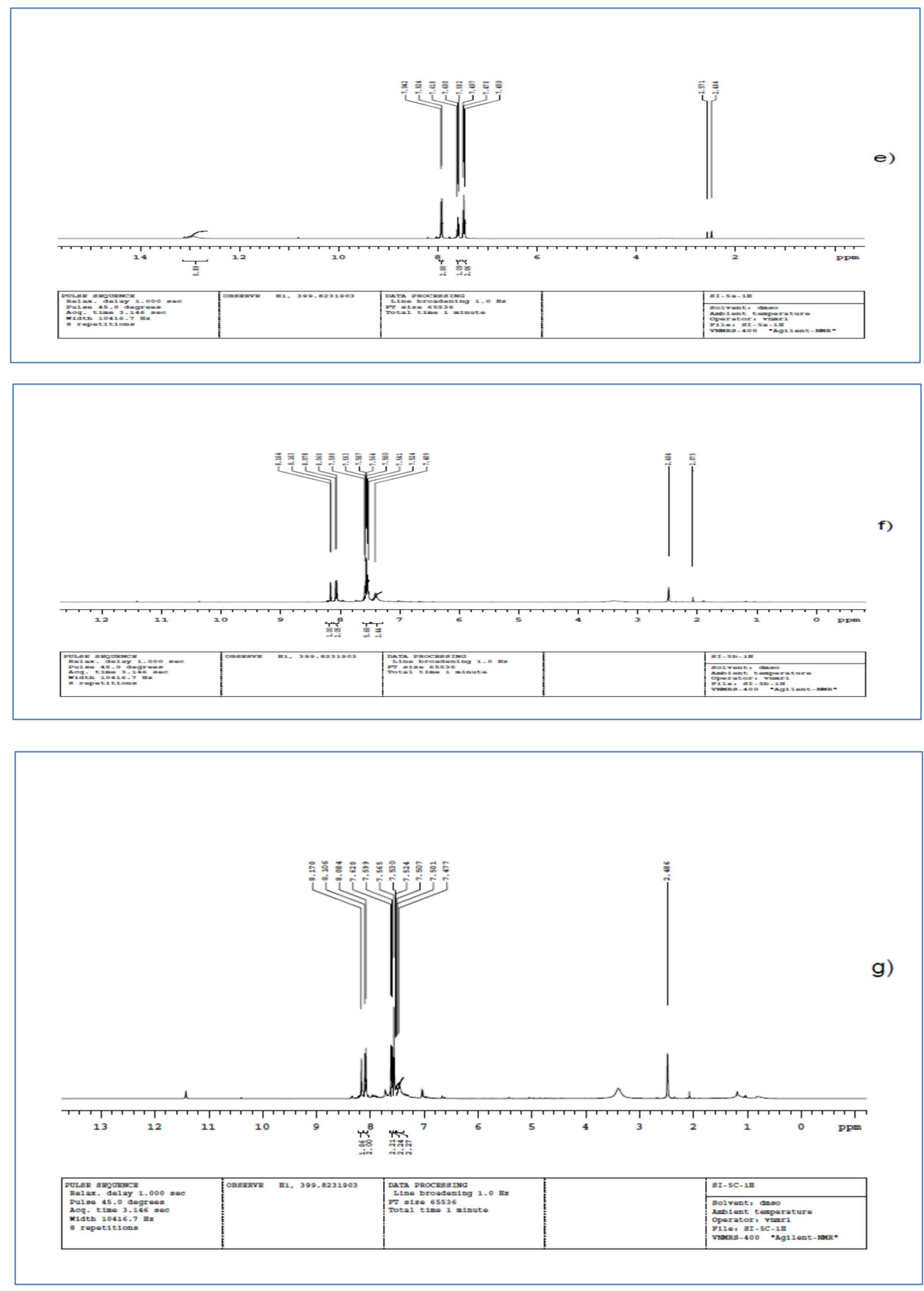

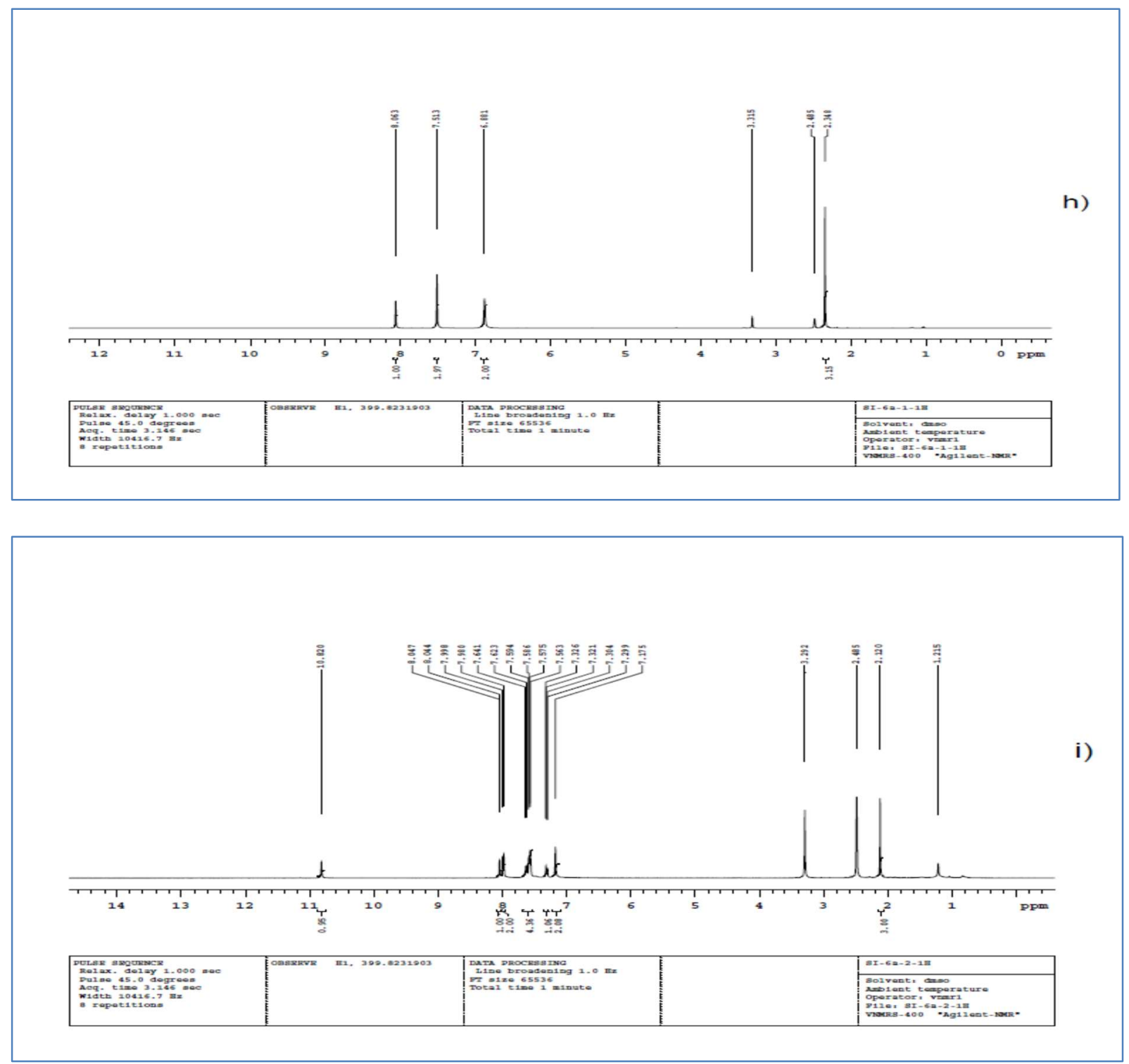

Fig.-1, ${ }^{1}$ HNMR Spectra of (a) Compound 3, (b) Compound 4, (c) Compound 5a, (d) Compound I 5b,

\section{Compound 5a}

(e) Compound 5c, (f) compound 5d, (g) Compound 5e, (h) Compound 6a

Obtained as a White solid; Yield: 55.52mg (90\%)IR $\left(\mathrm{cm}^{-1}, \mathrm{KBr}\right): 1641(\mathrm{C}=\mathrm{O}), 3357\left(\mathrm{NH}_{2}\right) ; \quad{ }^{1} \mathrm{H}-\mathrm{NMR}$ (DMSO-d6, $400 \mathrm{MHz}): \delta$ 2.4-2.6(d, $\mathrm{CH}=\mathrm{CH}, 2 \mathrm{H}) .7 .35\left(\mathrm{~s}, \mathrm{NH}_{2}, 2 \mathrm{H}\right), 7.58-7.66(\mathrm{~m}, \mathrm{Ar}-\mathrm{H}, 7 \mathrm{H})$. Mass $(\mathrm{m} / \mathrm{z})\left(\mathrm{M}^{+}\right): 246$.

\section{Compound 5b}

Obtained as a White solid; IR $\left(\mathrm{cm}^{-1}, \mathrm{KBr}\right): 1633(\mathrm{C}=\mathrm{O}), 3132\left(\mathrm{NH}_{2}\right) ;{ }^{1} \mathrm{H}-\mathrm{NMR}(\mathrm{DMSO}-\mathrm{d} 6,400 \mathrm{MHz}): \delta$ 2.6(s, C=CH, 1H), $2.1(\mathrm{~s}, \mathrm{CH} 3,3 \mathrm{H}), 7.35\left(\mathrm{~s}, \mathrm{NH}_{2}, 2 \mathrm{H}\right), 7.59-7.63(\mathrm{~m}, \mathrm{Ar}-\mathrm{H}, 7 \mathrm{H}), \mathrm{Mass}(\mathrm{m} / \mathrm{z})\left(\mathrm{M}^{+}\right)$: 299.

\section{Compound 5c}

Obtained as a White solid; IR ( $\left.\mathrm{cm}^{-1}, \mathrm{KBr}\right): 1658(\mathrm{C}=\mathrm{O}), 3208\left(\mathrm{NH}_{2}\right) ; \quad{ }^{1} \mathrm{H}-\mathrm{NMR}(\mathrm{DMSO}-\mathrm{d} 6,400 \mathrm{MHz})$ : $\delta$ 2.4-2.6(d, $\mathrm{CH}=\mathrm{CH}, 2 \mathrm{H}), 6.91\left(\mathrm{~s}, \mathrm{NH}_{2}, 2 \mathrm{H}\right), 7.26-7.49(\mathrm{~m}, \mathrm{Ar}-\mathrm{H}, 7 \mathrm{H}) . \operatorname{Mass}(\mathrm{m} / \mathrm{z})\left(\mathrm{M}^{+}\right): 332$.

\section{Compound 5d}

Obtained as a White solid, IR $\left(\mathrm{cm}^{-1}, \mathrm{KBr}\right): 1616(\mathrm{C}=\mathrm{O}), 3174\left(\mathrm{NH}_{2}\right) ;{ }^{1} \mathrm{H}-\mathrm{NMR}$ (DMSO-d6, $\left.400 \mathrm{MHz}\right): \delta$ 2.4-2.6(d, $\mathrm{CH}=\mathrm{CH}, 2 \mathrm{H}), 7.31\left(\mathrm{~s}, \mathrm{NH}_{2}, 2 \mathrm{H}\right), 7.42-7.46(\mathrm{~m}, \mathrm{Ar}-\mathrm{H}, 7 \mathrm{H})$. Mass (m/z) $\left(\mathrm{M}^{+}\right): 332$. 


\section{RASĀYAN J. Chem.}

Vol. 14 | No. 3 |1699-1710| July - September | 2021

\section{Compound 5e}

Obtained as a White solid; IR $\left(\mathrm{cm}^{-1}, \mathrm{KBr} 1633(\mathrm{C}=\mathrm{O}), 3316\left(\mathrm{NH}_{2}\right) ;{ }^{1} \mathrm{H}-\mathrm{NMR}\right.$ (DMSO-d6, $\left.400 \mathrm{MHz}\right): \delta$ 2.4-2.6( d, $\mathrm{CH}=\mathrm{CH}, 2 \mathrm{H}), \quad 3.80\left(\mathrm{~s}, \mathrm{OCH}_{3}, 3 \mathrm{H}\right), 6.88\left(\mathrm{~s}, \mathrm{NH}_{2}, 2 \mathrm{H}\right), 7.37-7.56(\mathrm{~m}, \mathrm{Ar}-\mathrm{H}, 7 \mathrm{H}) . \mathrm{Mass}(\mathrm{m} / \mathrm{z})$ $\left(\mathrm{M}^{+}\right): 328$.

\section{Compound 6a}

Obtained as a White solid; IR $\left(\mathrm{cm}^{-1}, \mathrm{KBr}\right): 1724(\mathrm{C}=\mathrm{O}), 3233\left(\mathrm{NH}_{2}\right) ;{ }^{1} \mathrm{H}-\mathrm{NMR}$ (DMSO-d6, $\left.400 \mathrm{MHz}\right): \delta$ 2.2-2.4(d, $\mathrm{CH}=\mathrm{CH}, 2 \mathrm{H}), 3.4(\mathrm{~s}, \mathrm{CH} 3,3 \mathrm{H}), 7.61(\mathrm{~s}, \mathrm{NH}, 1 \mathrm{H}), 7.75-7.77(\mathrm{~m}, \mathrm{Ar}-\mathrm{H}, 7 \mathrm{H}), \mathrm{Mass}(\mathrm{m} / \mathrm{z})\left(\mathrm{M}^{+}\right)$: 383.

\section{FTIR Spectra}
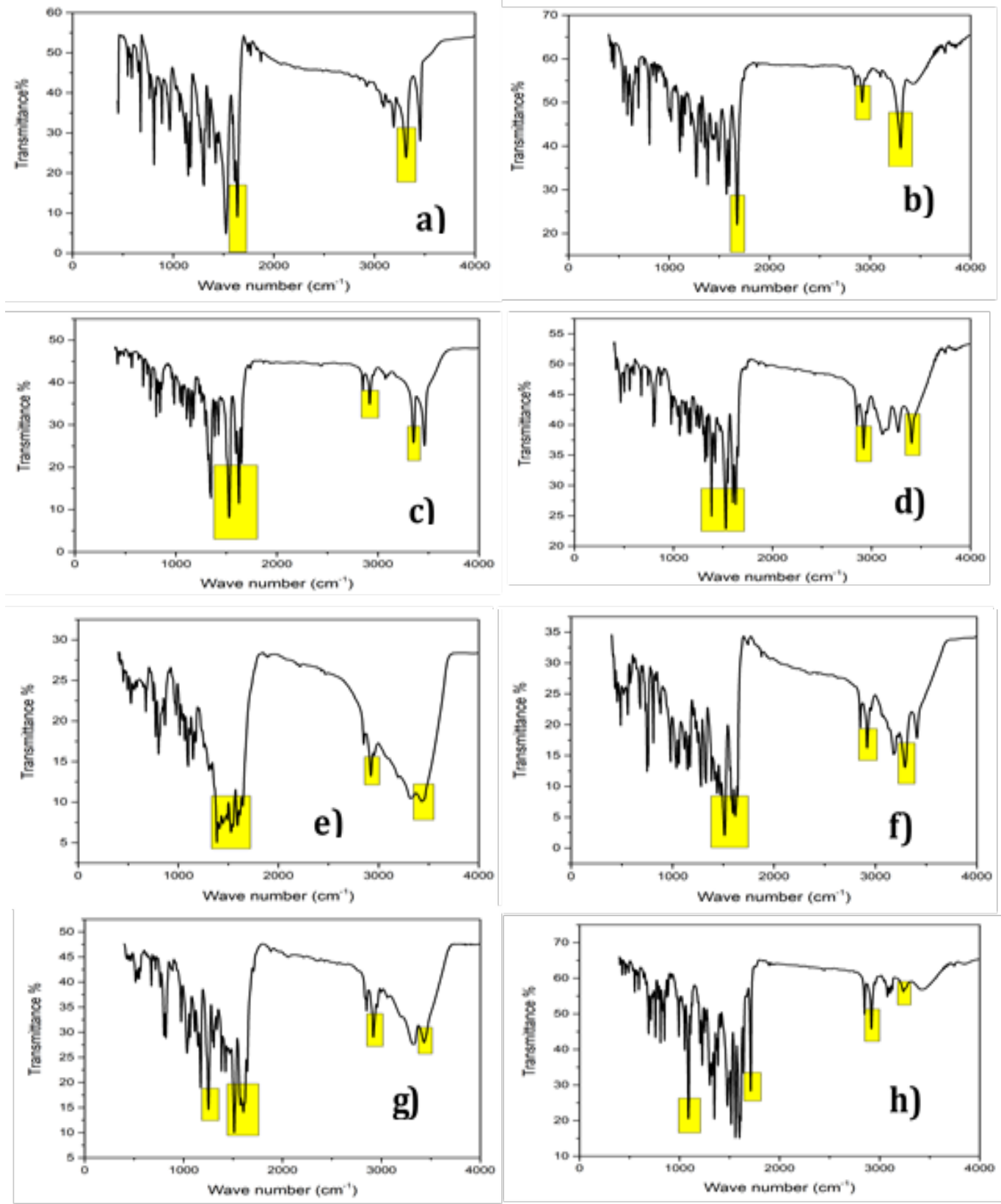

Fig.-2: FTIR Spectra of (a) Compound 3 , (b) Compound 4, (c) Compound 5a, (d) Compound I 5b, (e) Compound 5c, (f) compound 5d, (g) Compound 5e, (h) Compound 6a 
RASĀYAN J. Chem.

Vol. 14 | No. 3 |1699-1710| July - September | 2021

\section{Mass Spectra}
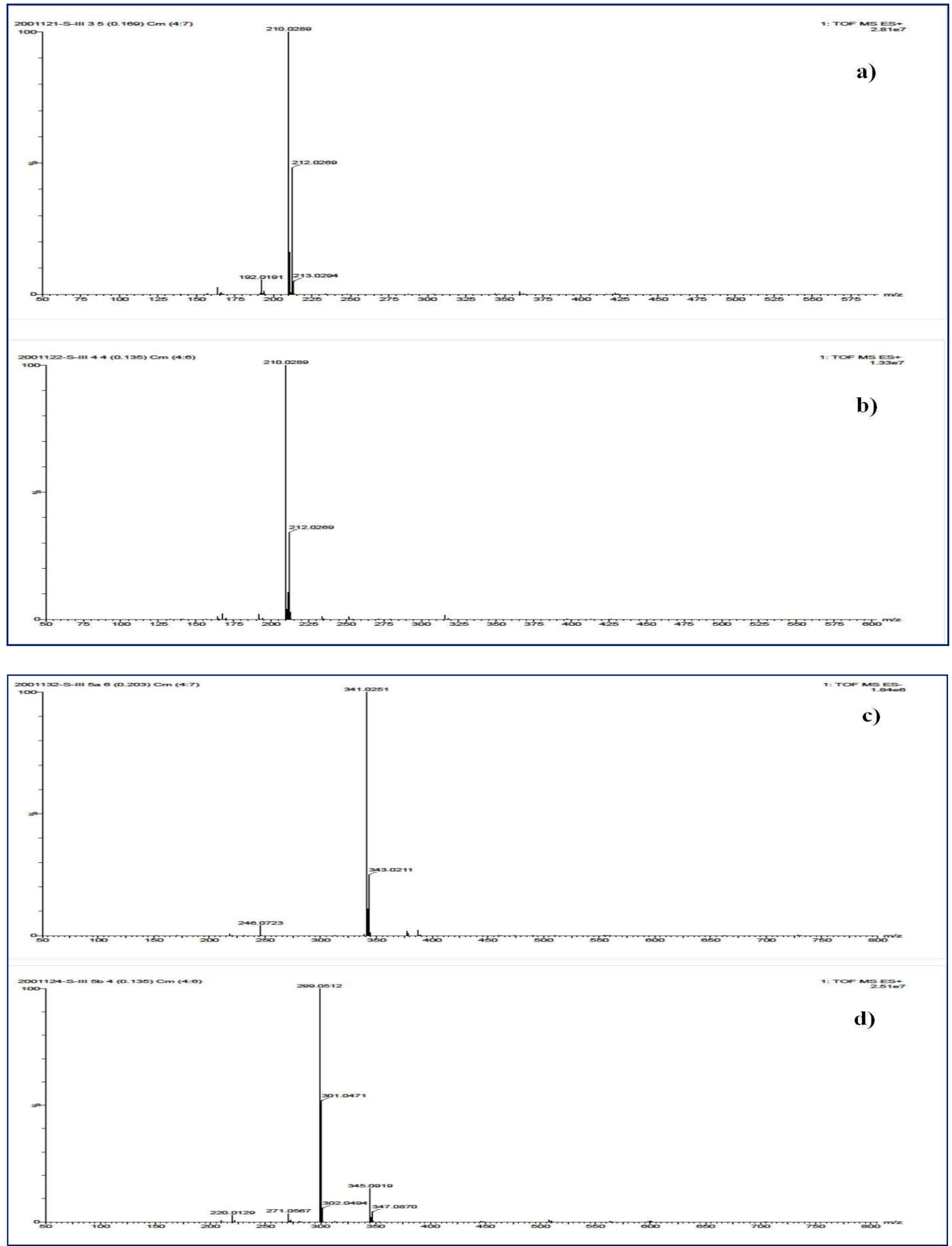
RASĀYAN J. Chem.

Vol. 14 | No. 3 |1699-1710| July - September | 2021

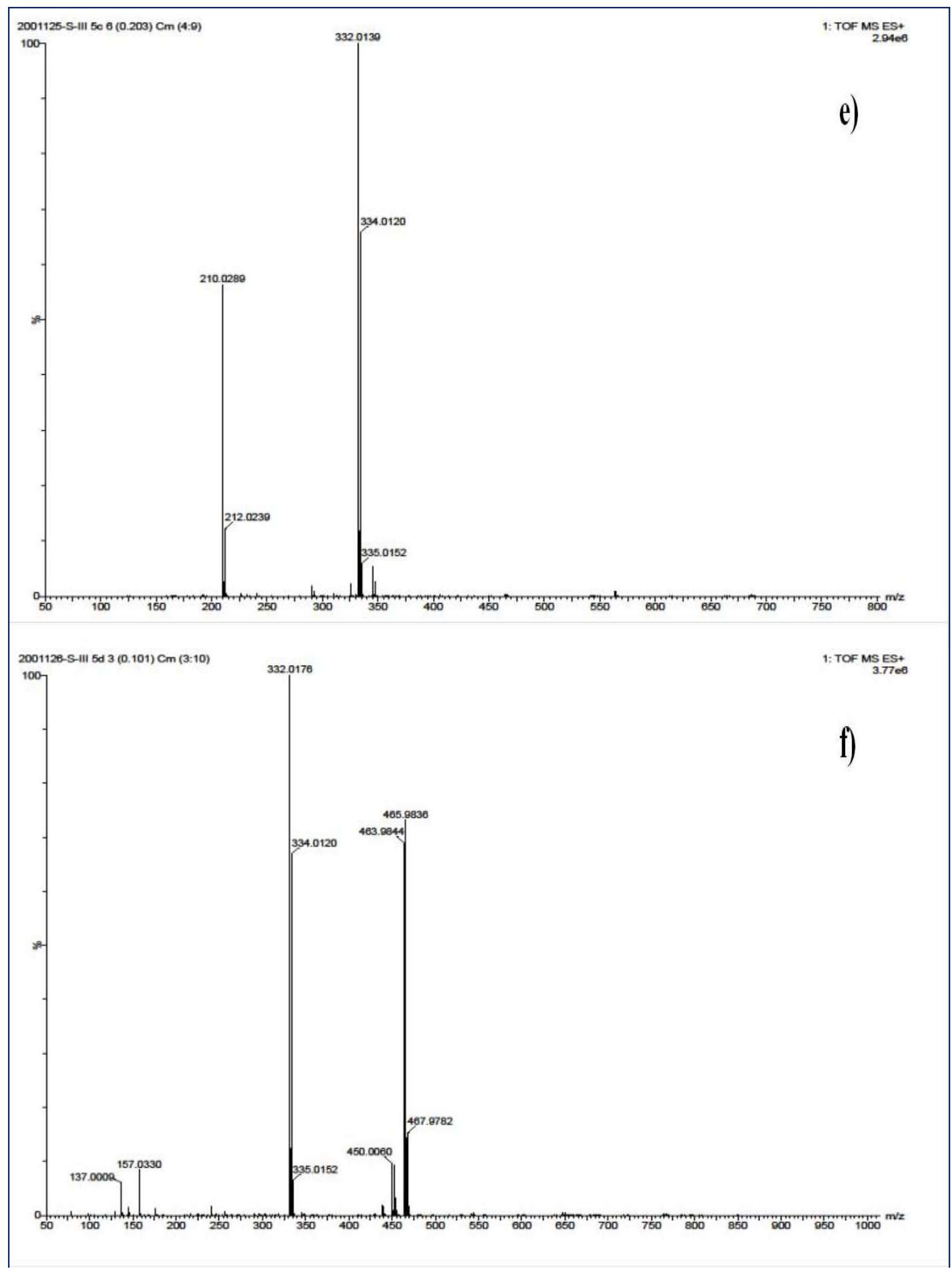


RASĀYAN J. Chem.

Vol. 14 | No. 3 |1699-1710| July - September | 2021

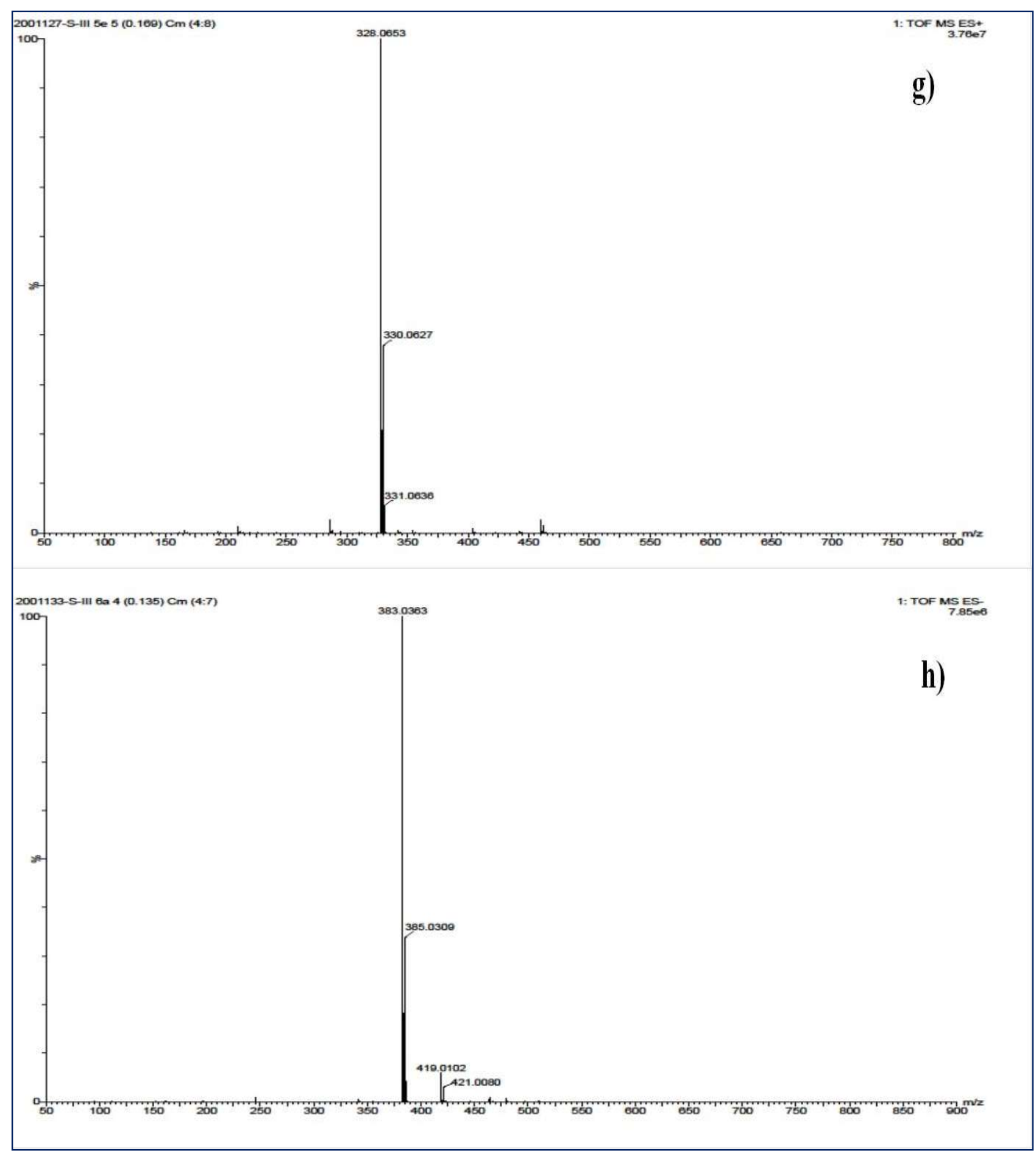

Fig.-3 Mass Spectra of (a) Compound 3, (b) Compound 4, (c) Compound 5a, (d) Compound I 5b,

\section{Biological Activity}

(e) Compound 5c, (f) compound 5d, (g) Compound 5e, (h) Compound 6a

The synthesized samples were analyzed for antibacterial properties against Staphylococcus aureus, Escherichia coli and Salmonella typhimurium. Further compounds were subjected to antifungal activities against Saccharomyces cerevisiae, and Aspergillus niger by dispersion method. he bacteria were grown in Muller-Hintonagar (Hi-media) plate $(370 \mathrm{C}, 24 \mathrm{~h})$ and the fungi were grown on Sabouraud dextrose agar (Hi-media) plates (26 0C, 48-72h). A loop of two to eight hours old bacterial/fungi was mixed in an inoculum in sterile distilled water. One-two drops of inoculums were spread on the plate of agar surface. 
RASĀYAN J. Chem.

Vol. 14 | No. 3 |1699-1710| July - September | 2021

Sterile discs (containing $100 \mu \mathrm{g}$ of the test sample) were incubated immediately. The activity was determined by measuring the diameter of zones of inhibition in $\mathrm{mm}$ diameter.

Table-2: Antibacterial Studies of prepared Compounds

\begin{tabular}{c|c|c|c}
\hline $\begin{array}{c}\text { Compound } \\
\text { name } 100 \mathrm{~g} / \mathrm{disc}\end{array}$ & Escherichia coli & Staphylococcus aureus & Salmonella typhimurium \\
\hline $\mathbf{3}$ & $06 \pm 0.02$ & $09 \pm 0.25$ & $04 \pm 0.45$ \\
\hline $\mathbf{4}$ & $10 \pm 0.08$ & $06 \pm 0.12$ & $08 \pm 0.42$ \\
\hline $\mathbf{5 a}$ & $05 \pm 0.05$ & $08 \pm 0.24$ & $03 \pm 0.18$ \\
\hline $\mathbf{5 b}$ & $10 \pm 0.07$ & $11 \pm 0.36$ & $08 \pm 0.12$ \\
\hline $\mathbf{5 c}$ & $17 \pm 0.45$ & $06 \pm 0.14$ & $07 \pm 0.35$ \\
\hline $\mathbf{5 d}$ & $10 \pm 0.08$ & $11 \pm 0.28$ & $09 \pm 0.58$ \\
\hline $\mathbf{5 e}$ & $07 \pm 0.04$ & $05 \pm 0.18$ & $08 \pm 0.15$ \\
\hline $\mathbf{6 a}$ & $12 \pm 0.1$ & $10 \pm 0.16$ & $06 \pm 0.25$ \\
\hline $\begin{array}{c}\text { Gentamicin } \\
\text { (Standard) }\end{array}$ & 20 & 20 & 20 \\
\hline
\end{tabular}

The antibacterial activity has been tabulated in Table-2. Compound $5 \mathrm{c}$ exhibited moderately high activity against Escherichia coli, compounds 4, 5b, 5d, and 6a showed moderate activity against the same organisms. The rest of the compounds were associated with low activity compared to the standard drug. Compounds 5b, 5d, 6a, and 3 exhibited moderate activities and the remainder showed low activity against Staphylococcus aureus. All the compounds synthesized exhibited low activity against Salmonella typhimurium.

Table-3: Antifungal Activity for Synthesis Compounds

\begin{tabular}{c|c|c}
\hline $\begin{array}{c}\text { Compound } \\
\text { name } 100 \mathrm{~g} / \mathrm{disc}\end{array}$ & Aspergillus niger & $\begin{array}{c}\text { Saccharomyces } \\
\text { cerevisiae }\end{array}$ \\
\hline $\mathbf{3}$ & $02 \pm 0.14$ & $02 \pm 0.06$ \\
\hline $\mathbf{4}$ & $05 \pm 0.41$ & $07 \pm 0.45$ \\
\hline $\mathbf{5 a}$ & $05 \pm 0.23$ & $02 \pm 0.26$ \\
\hline $\mathbf{5 b}$ & $08 \pm 0.15$ & $05 \pm 0.09$ \\
\hline $\mathbf{5 c}$ & $07 \pm 0.23$ & $06 \pm 0.32$ \\
\hline $\mathbf{5 d}$ & $06 \pm 0.11$ & $05 \pm 0.21$ \\
\hline $\mathbf{5 e}$ & $10 \pm 0.19$ & $10 \pm 0.68$ \\
\hline $\mathbf{6 a}$ & $10 \pm 0.25$ & $09 \pm 0.51$ \\
\hline $\begin{array}{c}\text { Acetin } \\
\text { (Standard) }\end{array}$ & 18 & 18 \\
\hline
\end{tabular}

The antifungal activity was tabulated in Table-3. Compounds $\mathbf{5 e}, \mathbf{6 a}$, and $\mathbf{5 b}$ showed moderate activity against the organism Aspergillus niger, compounds $5 \mathbf{e}$ and $\mathbf{6 a}$ showed moderate activity against Saccharomyces cerevisiae. The remainder of the compounds synthesized is low-active compared to standard drugs for both organisms.

\section{CONCLUSION}

The newly synthesized compounds were characterized by their IR, ${ }^{1} \mathrm{HNMR}$, and Mass Spectral data. The data is in concurrent with the assigned structures. All the compounds synthesized in the present investigation were screened for their antibacterial and antifungal activity. Compounds have shown encouraging antibacterial and antifungal activity against the selected organisms and some were comparable with standard drugs used.

\section{ACKNOWLEDGEMENT}

The authors express sincere thanks to the Dept. of Chemistry/Industrial chemistry, VSK University, for providing lab facility during the present work. We also extend our thanks to Dr. Shivakumar Hugar of BLDE College of Pharmacy, Vijayapura (Karnataka) for helping in getting biological activity screening. 


\section{RASĀYAN J. Chem.}

Vol. 14 | No. 3 |1699-1710| July - September | 2021

\section{REFERENCES}

1. Z. Nowakowska, European Journal of Medicinal Chemistry 42, 125(2007). https://doi.org/10.1016/j.ejmech.2006.09.019

2. A. Agarwal, K. Srivastava and S. K. Puri, Prem M. S. Chauhan, Bioorganic \& Medicinal Chemistry Letters, 15(12), 3133(2005). https://doi.org/10.1016/j.bmcl.2005.04.011

3. P.M. Sivakumar, S. Ganesan, P. Veluchamy, Mukesh Doble, Chemical Biology \& Drug Design, 76, 407(2010), https://doi.org/10.1111/j.1747-0285.2010.01020.x

4. J.C. Aponte, M. Verastegui, E. Malaga, Mirko Zimic, Miguel Quiliano, Abraham J. Vaisberg, Robert H. Gilman and Gerald B. Hammond, Journal of Medicinal Chemistry, 51, 6230(2008). https://doi.org/10.1021/jm800812k

5. Afzal Shah, Asad M. Khan ,Rumana Qureshi, Farzana L. Ansari, Muhammad F. Nazar and Syed S. Shah, International Journal of Molecular Sciences, 9, 1424(2008), https://doi.org/10.3390/ijms9081424

6. Jérôme Quintin, Julie Desrivot, Sylviane Thoret, Patrick LeMenez, Thierry Cresteil, Guy Lewin $\begin{array}{lllll}\text { Bioorganic \& Medicinal Chemistry Letters, 19(1), } & \text { 167(2009). }\end{array}$ https://doi.org/10.1016/j.bmcl.2008.10.126

7. Srinivas K. Kumar, Erin Hager, Catherine Pettit, Hallur Gurulingappa, Nancy E. Davidson, and Saeed R. Khan, Journal of Medicinal Chemistry, 46(14), 2813(2003). https://doi.org/10.1021/jm030213+

8. J. R. Dimmock, D. W. Elias, M. A. Beazely, N. M. Kandepu, Current Medicinal Chemistry, 6(12), 1125(1999).

9. J.H. Wu, X.H. Wang, Y.H. Yi, K.H. Lee, Bioorganic \& Medicinal Chemistry Letters, 13, 1813 (2003), https://doi.org/10.1016/s0960-894x(03)00197-5

10. M.L. Go, X. Wu, X.L. Liu, Current. Medicinal Chemistry, 12, 483(2005). https://doi.org/10.2174/0929867053363153

11. V. Ugale, H. Patel, B. Patel, and S. Bari, Arabian Journal of Chemistry, S389-S396(2012), https://doi.org/10.1016/j.arabjc.2012.09.011

12. D. Cos,kun and M. Ahmedzade, Synthetic Communications, 38(21), 3613(2008), https://doi.org/10.1080/00397910802178997

13. Manjunatha Harihara Mathada, H. M. Naveenakumari, and K. M. Basavaraja, Journal Applicable Chemistry, 8(1), 165(2019).

14. K. M. Basavaraja, Manjunatha Harihara Mathada, H. M. Naveena Kumari, Mahesh Kumar, K. T. Sudha, Asian Journal of Research in Chemistry and Pharmaceutical Science, 7(1), 233(2019).

[RJC-6422/2021] 